\title{
BIFURCATION AND SIZE EFFECT IN A VISCOELASTIC NON-LOCAL DAMAGEABLE CONTINUUM
}

\author{
Th. Baxevanis, G. Pijaudier-Cabot and F. Dufour \\ R\&DO, Institut de recherches en Genie Civil \\ Ecole Centrale de Nantes, 1 rue de la Noe \\ BP 92101, F-44321 Nantes cedex 3, FRANCE \\ theocharis@tem.uoc.gr
}

The growth of microcracks in progressively fracturing rate-independent materials is a fairly distributed process which is associated with stable material response. However, for certain stress trajectories such as tertiary creep, a different deformation mode may prevail, consisting of formation of discrete failure planes (macrocracks). In the latter case, the mechanical response as observed on the macroscale becomes, in general, unstable. The inception of such a localized mode may be considered as a bifurcation problem related to the loss of positive definiteness of the tangent material stiffness operator governing the homogeneous deformation (Rudnicki and Rice [11]). In mathematical terms, the elliptic character of the set of governing differential equations, in quasi-static problems, is lost (ill-posedness of the related boundary value problem). This result was derived for the linearized rate equation problem, considering what is commonly denoted as a linear comparison solid (Hill [5]).

In common practice, it is usually assumed that for low stress levels linear viscoelasticity takes place and that the instantaneous mechanical behavior is elastic. Viscoelasticity of the material causes strong time dependence of fracture growth. Bazant and Gettu [2] conducted size effect tests on geometrically similar specimens loaded at different rates and observed that the slower the loading rate, the more brittle the response. Pijaudier-Cabot et al [9] performed also a series of three point bending beam tests (notched specimens) applying a sustained load for 3 months up to 85 percent of the maximum load prior to the fracture test. They arrived to a similar conclusion, namely, that upon size effect tests, the specimens subjected to creep exhibited a more brittle response compared to the ones that were not. This property yields a shift to the right of the data set on the size effect plot.

For high stress levels the linearity hypothesis fails. Nevertheless, creep cannot be captured merely by nonlinear generalization of viscoelastic stress-strain relation (Bazant [3]). The experimental evidence associates creep strains at high stress levels to microcracking nucleation and growth - cracks grow and interact with visco-elasticity - (Rüsch [12] was the first one to conduct such experiments). Some experimental and analytical results concerning non-linear creep can be found in the literature (see among others Bazant [1], Bazant and Gettu [2], Mazzotti and Savoia [7]). The coupling of the mechanical behaviour and the visco-elastic behaviour of the material assumed as a continuum, and its nonlinear instantaneous behaviour has been tackled by many authors using a linear creep model (viscoelastic behaviour) coupled with a plasticity model (De Borst et al [4]), or a smeared crack model (Rots et al [10]), or a damage model (Mazzotti and Savoia [7]) (nonlinear instantaneous behaviour).

The aim of this paper is to investigate the localization properties of a rate-independent material described by a non-local damage-based constitutive relation coupled with a creep relation of the Kelvin type. It is proved that the coupled model preserves the properties of localization limiters as they were pointed out in the paper of Pijaudier-Cabot and Benallal [8] and reproduces the experimentally observed rate-effects of creep. Namely, the minimum wavelength of the localization modes can not be zero in accordance with energy considerations and the material 
response due to creep becomes more brittle, at least under a certain condition on the evolution of the yield function of damage. Moreover, the normal $\mathbf{n}$ to the localization band depends only upon the elastic state of strain at the onset of bifurcation and a shift on the size effect plot occurs towards the strength criterion when the material parameter $(01)$, which is the fraction of creep release energy which contributes to damage, is decreasing. The motivation for introducing this parameter into the constitutive law is that for low stress levels although creep strain can be large (even larger than that corresponding to peak stress for short term loading) there is no significant variation of the elastic modulus; that is no significant damage (Mazzotti and Savoia [6]). The proposed model fails to predict creep rate-effects when damage is supposed, as it is common practice, to be driven solely from the elastic release energy $(=0)$.

\section{References}

1. Bazant Z.P., Mathematical modeling of creep and shrinkage of concrete, John Wiley \& Sons Ltd, 1988.

2. Bazant, Z.P. and Gettu, R., ACI Materials Journal, vol 89, 456-468, 1992.

3. Bazant, Z.P., In Proceedings of the 5th Int. RILEM Symp. on Creep and Shrinkage of Concrete, edited by Z.P. Bazant and I. Carol, Barcelona, E\&FN Spon, London, 291-307, 1993.

4. De Borst, R., Van Den Boogaard, A. H. And Sluys, L. J., In the Proccedings of the 5th Int. RILEM Symp. on Creep and Shrinkage of Concrete, edited by Z.P. Bazant and I. Carol, Barcelona, E\&FN Spon, London, 309-325, 1993.

5. Hill, R., J. Mech. Phys. Solids, vol 7, 209-225, 1959.

6. Mazzotti, C. and Savoia, M., In Proceedings of Concreep 6, edited by Z.P. Bazant, F.-J. Ulm and F.H. Whitmann, Cambridge, England, Elsevier, Amsterdam, 259-264, 2001.

7. Mazzotti, C. and Savoia, M., J. Engrg. Mech. ASCE, vol 129, 1065-1075, 2003.

8. Pijaudier-Cabot, G. and Benallal, A., Int. J. Solids Structures, vol 30, 1761-1775, 1993.

9. Pijaudier-Cabot, G., Omar, M., Loukili, A. and Le Pape, Y., In Proccedings of ICF 11, Torino, March 2005.

10. Rots, J. G., Dissertation, Delft University of Technology, Delft, the Netherlands.

11. Rudnicki, J. and Rice, J. R., J. Mech. Phys. Solids, vol 23, 371-394, 1975.

12. Rusch, H., $A C I j$., vol 57 (1), 1-28, 1960. 